\title{
The effects of transport stress on the behaviour and adrenocortical activity of the black-and-white ruffed lemur (Varecia variegata)
}

\author{
Martina Volfová1, Zuzana Machovcová1, Franz Schwarzenberger², Eva Voslářová1, \\ Iveta Bedáňová1, Vladimír Večerek ${ }^{1}$
}

\begin{abstract}
${ }^{1}$ University of Veterinary and Pharmaceutical Sciences Brno, Faculty of Veterinary Hygiene and Ecology, Department of Animal Protection, Welfare and Behaviour, Brno, Czech Republic

${ }^{2}$ University of Veterinary Medicine Vienna, Department of Biomedical Sciences, Austria
\end{abstract}

Received November 19, 2018

Accepted February 12, 2019

\begin{abstract}
The aim of this study was to assess behavioural and glucocorticoid changes in blackand-white ruffed lemur females transported for breeding purposes between Czech ZOOs. The frequency of the presented behaviour elements was recorded via direct observation. The faecal samples were collected from the floor and subsequently the faecal glucocorticoid values were determined using specifically designed assays for faecal glucocorticoid metabolite (FGM) analysis. While the frequency of certain comfort behaviour patterns decreased $(P<0.05)$ in response to transport and change of the environment, grooming, resting, sleeping as well as playful behaviour (chasing, climbing) were not affected $(P>0.05)$. No changes were found in the frequency of behaviour connected to metabolic events with the exception of defecation. Lemur females defecated more frequently $(P<0.05)$ in their new environment. The frequency of neither fear nor exploratory behaviour differed $(P>0.05)$ before and after transport. The positive effect of the novelty was shown by the reduction $(P<0.01)$ of behavioural signs of frustration and stereotypical behaviour after transport. In response to transport, a significant $(P<0.05)$ increase of FGM levels was found the second day after the transport, reaching peak levels in the morning and starting to decline to baseline levels in the afternoon. The results of both behaviour and adrenocortical activity analysis suggest that despite some presented changes in the monitored indices prior and after transport, the transportation under the studied conditions presented only a mild stressor with limited behavioural and glucocorticoid responses.
\end{abstract}

ZOO animals, ethogram, glucocorticoids, faecal samples, ELISA

Black-and-white ruffed lemurs (Varecia variegata) inhabit lowland to mid-altitude rain forests in eastern Madagascar. Due to habitat loss, logging, mining and hunting, their wild populations are decreasing. Since 2008 , the species has been listed as critically endangered by the IUCN Red List of Threatened Species (IUCN 2018). They are also listed in Appendix I of CITES (CITES 2017), belonging among the most endangered among CITES-listed animals and plants. Considering their conservation status, black-and-white ruffed lemurs are one of the priority species in terms of developing both in situ and ex situ conservation programs (King et al. 2013; Schwitzer et al. 2013).

ZOOs play an important role to prevent the extinction of species in nature. Captive animals can serve as a resource for re-establishing endangered populations and maintaining genetic diversity (Dembiec et al. 2004). Transportation is often necessary for breeding purposes but presents a strong stress factor for the animals. For studying changes in the behaviour and physiology during and after the transport, the use of a combination of behavioural and physiological measurements will provide the best overall measurement of animal discomfort (Grandin 1997).

The behavioural observation is one of the important methods to assess the 
well-being of captive bred animals. Detailed observation of animals in the wild is key to understanding their behaviour. In the past, numerous published ethograms described the detailed behaviour of cercopithecine monkeys and apes (Pereira et al. 1988). However, no information on lemurs and other prosimian primates was available until the study by Pereira et al. (1988) was published. The authors provided a detailed description of all behaviour patterns observed in a semifree-ranging group of black-and-white ruffed lemurs (Varecia variegata variegata). Besides the behaviour observations, also the stress response to environmental changes can be analysed with the use of laboratory analytical methods. The monitoring of endocrine markers is a well-known method for measuring stress. While blood collection has considerable limitations, non-invasively collected samples such as urine, saliva, hair or faeces can be obtained without any limitations or disturbance of the observed animal. The methodology of non-invasive monitoring of adrenocortical activity has been described e.g. by Schwarzenberger and Brown (2013), Malikova et al. (2018), and others. Behringer and Deschner (2017) summarized the achievements in non-invasive monitoring of physiological markers in primates.

The aim of this study was to assess behavioural and glucocorticoid responses to stress related to transport in two black-and-white ruffed lemurs. This study did not differentiate between the single effects of several stressors affecting animals during their transfer (handling, floor space restrictions, change of the environment, change of daily routine, etc.).

\section{Materials and Methods}

The study animals were two females of black-and-white ruffed lemur (Varecia variegata). The females were siblings born on $13^{\text {th }}$ May 2015 in the ZOO in Olomouc where they were housed together with their parents since their birth. Female 1 was the dominant one, distinguished by white colour on the tail. Female 2 was submissive and had an all-black tail.

For breeding purposes, the females (aged 17 months) were transported from the Olomouc ZOO to the Brno ZOO on $6^{\text {th }}$ October 2016. During the transport, the females were placed separately in two pet transport crates but were in visual, olfactory and auditory contact. The transport took approximately three hours (distance travelled $202 \mathrm{~km})$.

The ethological observation started three days prior to the transport and continued until four days after the transport. The frequency of the presented behaviour elements was recorded into pre-prepared ethogram sheets every $2 \mathrm{~min}$ for $2 \mathrm{~h}$ each day (from 10:00 to 11:00 $\mathrm{h}$ and from 14:00 to 15:00 h). The recorded behaviour elements were counted in total from both females three days prior to transport (i.e. six values for frequency of the presented behaviour elements were gained in total) and four days after the transport (i.e. eight values for frequency of the presented behaviour elements were gained in total).

Furthermore, non-invasive monitoring of adrenocortical activity in response to transport was carried out. The faecal samples were collected from the floor twice a day while avoiding urine and water contamination, starting one day prior to transport and ending two days after transport excluding the transport day (no samples were available). The sampling intervals ranged between 10:00 to 12:00 $\mathrm{h}$ and 13:00 to 15:00 h. The faecal samples were placed in plastic bags within one hour of defecation, marked and directly transferred to a freezer at $-20{ }^{\circ} \mathrm{C}$. Sample processing and faecal glucocorticoid analysis were performed at the University of Veterinary Medicine, Vienna, Austria. Samples for endocrine monitoring were extracted by vortexing a

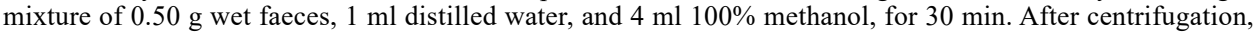
the obtained extracts were diluted in assay buffer. Faecal immunoreactive glucocorticoid metabolite (FGM) concentrations were analysed using enzyme immunoassays (EIAs) developed and courteously provided by Dr. R. Palme, Vienna. The polyclonal antibodies used in the EIAs were produced in rabbits using immunogens against 11-oxoaetiocholanolone I (72a, measuring 11,17-dioxo-androstanes; 11,17-DOA) (Palme and Möstl 1997), 11-oxoaetiocholanolone II (72T, measuring FGM with a 5 $\beta$-3 $\alpha$-ol-11-one structure) (Möstl et al. 2002) and 11 -hydroxyaetiocholanolone (69a, measuring FGM with a $5 \beta-3 \alpha, 11 \beta$-diol structure) (Frigerio et al. 2004).

The results were analysed using the statistical package Unistat 5.6. (Unistat Ltd., London, England). Because of non-normality of the data (Shapiro-Wilk test), non-parametrical methods were used for their analysis. The median of frequencies of the behaviour elements presented before and after the transport was calculated and subsequently compared by means of the Mann-Whitney U test (Zar 1999). Faecal glucocorticoide metabolite concentrations measured one day prior to the transport, one day, and two days after the transport were compared by means of Kruskall-Wallis ANOVA. A $P$-value $<0.05$ was considered significant. 


\section{Results}

The observed behaviour elements and their frequencies are presented in Table 1. Prior to the transport, some comfort behaviour patterns were observed significantly more frequently than after the transport, namely a higher frequency of sunning $(P<0.05)$, scratching $(P<0.05)$, stretching $(P<0.01)$, and allogrooming $(P<0.01)$ was found in both females. However, the frequency of other comfort behaviour patterns (grooming, resting, sleeping) and playful behaviour (chasing, climbing) were not affected $(P>0.05)$ by either the transport or the change of the environment. No changes were found in the frequency of behaviour connected to metabolic events (foraging, feeding, drinking, urination) with the exception of defecation. The lemurs defecated more frequently $(P<0.05)$ in their new environment. The frequency of fear or exploratory behaviour did not differ $(P>0.05)$ before and after the transport. Interestingly, higher $(P<0.01)$ frequency of behaviour signs of frustration and stereotypical behaviour was observed in the original housing in comparison to the new environment.

Table 1. Observed behaviour elements and their frequency in female lemurs before and after transport.

\begin{tabular}{|c|c|c|c|}
\hline \multirow{2}{*}{ Behaviour elements } & \multicolumn{2}{|c|}{ Median of frequency } & \multirow{2}{*}{$\begin{array}{c}\text { Significance - } P \\
\text { (Mann-Whitney U test) }\end{array}$} \\
\hline & Before transport & After transport & \\
\hline Grooming & 6.0 & 4.5 & N.S. \\
\hline Sunning & 2.0 & 0.0 & 0.019 \\
\hline Stretching & 4.0 & 2.0 & 0.004 \\
\hline Scratching & 4.0 & 2.2 & 0.029 \\
\hline Resting & 24.0 & 24.0 & N.S. \\
\hline Sleeping & 0.0 & 0.0 & N.S. \\
\hline Allogrooming & 5.5 & 2.0 & 0.004 \\
\hline Drinking & 2.0 & 1.0 & N.S. \\
\hline Feeding & 9.5 & 8.5 & N.S. \\
\hline Foraging & 5.0 & 4.5 & N.S. \\
\hline Defecation & 2.0 & 3.5 & 0.023 \\
\hline Urination & 1.0 & 1.0 & N.S. \\
\hline Coprophagy & 1.0 & 0.0 & N.S. \\
\hline Vocalization & 1.0 & 2.5 & N.S. \\
\hline Exploration & 28.0 & 36.5 & N.S. \\
\hline Chasing & 5.0 & 1.5 & N.S. \\
\hline Climbing & 11.0 & 4.0 & N.S. \\
\hline Aggression & 0.0 & 0.0 & N.S. \\
\hline Fear & 0.0 & 1.5 & N.S. \\
\hline Frustration & 4.0 & 0.0 & 0.002 \\
\hline Stereotypy & 1.0 & 0.0 & 0.003 \\
\hline
\end{tabular}

N.S. $=$ not significant $(P>0.05)$

Faecal glucocorticoide metabolite concentrations one day prior to and one day after the transport did not differ $(P>0.05)$. A significant $(P<0.05)$ increase in FGM levels was seen two days after the transport, reaching peak levels in all three EIAs in the morning and starting to decline to baseline levels in the afternoon (Fig. 1). While comparisons between FGM levels one day and two days after the transport revealed significant differences in all three EIAs tested, comparing FGM levels one day before and two days after the transport, a significant $(P<0.05)$ difference 
was found only in two of the three assays tested (11-oxoaetiocholanolone II, $11 \beta$-hydroxyaetiocholanolone).

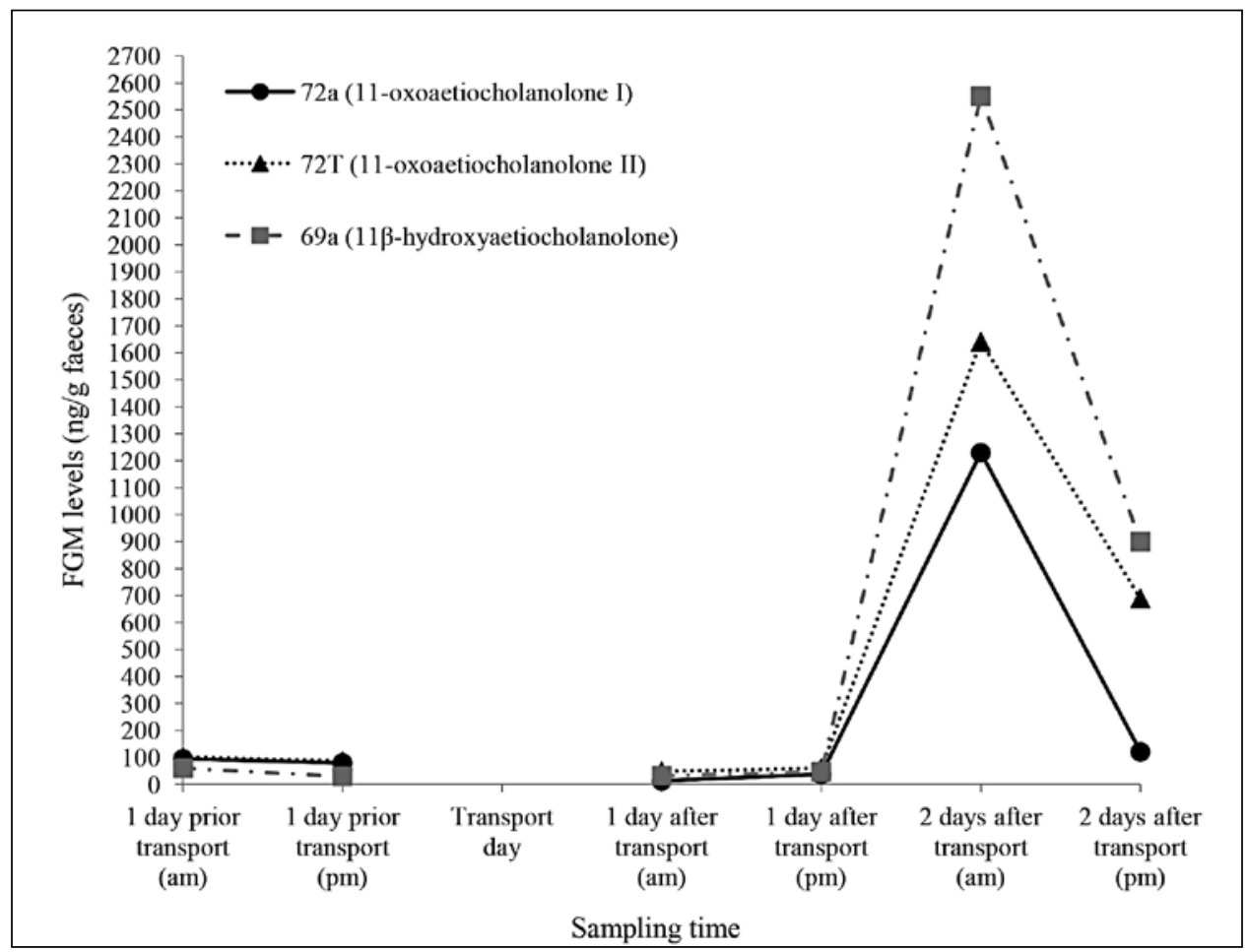

Fig. 1. Faecal glucocorticoide metabolite (FGM) concentrations in female lemurs before and after transport.

\section{Discussion}

The physiological and behavioural reactions in animals are controlled by a complex interaction of genetic factors and previous experiences (Grandin 1997). The extent of the impact is dependent upon the type and duration of stress, as well as the animals' past experiences. Since the monitored black-and-white ruffed female lemurs had never experienced any change of environment nor have they been transported before, a major stress response was expected. However, the transport was inevitable as the two females had to be removed from their native group before reaching sexual maturity. It is a routine practice in captive breeding that simulates animals leaving their birth groups before reaching sexual maturity in the wild as a natural prevention from inbreeding. Reproductive maturity in captive black-and-white ruffed lemur is reached at around 18-20 months of age (Porton 1989). Thus, at 16 months of age, the monitored females were ready to leave their birth group. A detailed description of the behavioural repertoire of free-ranging Varecia variegata was reported by Pereira et al. (1988). The social structure of wild ruffed lemurs is described as a multi-male/multi-female fission-fusion social organization (Vas ey 2006) and their activity is cathemeral according to recent studies (cathemerality, or activity throughout the 24-h cycle, is relatively common among e.g. ring-tailed lemurs and ruffed lemurs) (Bray et al. 2016). 
In general, the response to stress in animals is usually manifested with various physiological and behavioural changes (e.g. fear can inhibit exploratory behaviour or stimulate flight or hiding behaviour). Stress may also result in abnormal behaviour patterns, changes in facial expressions, position of ears, and increased respiration and pulse rate (Dembiec et al. 2004). An increase in body temperature (Brundige et al. 1998) and depressed immune response (Baker and Gemmell 1999) have been documented as effects of transportation stress. Activation of the hypothalamic-pituitary-adrenal axis with an elevation of circulating glucocorticoid levels is a typical marker of stress in animals (Sapolsky et al. 2000), which has been documented also in many studies in primates (Whitten et al. 1998; Heistermann et al. 2006; Clark et al. 2011; Pirovino et al. 2011; Weingrill et al. 2011; Shutt et al. 2012; Hämäläinen et al. 2014; Keller et al. 2014; Rangel-Negrín et al. 2014; Takeshita et al. 2018). Pride (2005) found the group size to affect stress in ring-tailed lemurs by measuring faecal cortisol concentrations with a radioimmunoassay (RIA). In other studies, stress effects of seasonality, sociality and reproduction in free-ranging ring-tailed lemurs were determined using a steroidextraction method to measure cortisol concentrations from female faeces using the RIA method (Cavigelli 1999) or faecal corticosterone levels determined by the RIA method (Starling et al. 2010). Similarly, Gould et al. (2005) found the effects of reproductive and social variables on faecal glucocorticoid levels (cortisol) in ring-tailed lemurs. Cavigelli et al. (2003) found an impact of the individual's position within the social hierarchy on glucocorticoid levels in ring-tailed lemurs.

In our study, the stress related to transport and the change of environment resulted in a decreased frequency of sunning, scratching, stretching, and allogrooming. The most prominent reduction was seen in sunning. Despite sunning being one of the typical behaviour patterns in ruffed lemurs, no sunning was recorded during the four days of observation after the transport. Normally, sunning is observed in captive ruffed lemurs during the whole year; in winter or when the sun is not shining, an infrared lamp is used. This behaviour is an important adaptation that augments chemical thermoregulation in the absence of dynamic insulating fur (Kelley et al. 2016). Common positions are upright sitting with the arms extended laterally, the head tilted back, frequently with the eyes closed, and supine lying (Pereira et al. 1988). However, the presence of other comfort behaviour patterns (grooming, resting, and sleeping) and playful behaviour (chasing, climbing) that are considered to be the indicators of positive welfare (Pereira et al. 1988) were not affected by the transport and change of the environment.

Similarly, no changes were found in the frequency of behaviour patterns connected to metabolic events (foraging, feeding, drinking, urination) with the exception of defecation. The lemurs defecated more frequently in their new environment. Despite the increased frequency of defecation, coprophagy was not observed after the transport. Coprophagy is often considered an indicator of negative welfare in captive primates (Campbell et al. 2000) and a number of hypotheses have been discussed to explain its occurrence including food deficiency, boredom, social stress, and medical issues (Krief et al. 2004).

Abnormal behaviour patterns such as stereotypy are often seen in animals kept in captivity. Tarou et al. (2005) studied stereotypy in prosimians and found $13.2 \%$ of prosimians at institutions accredited by the Association of Zoos and Aquariums (AZA) to exhibit some form of stereotypic behaviour. Individuals of the genus Varecia and Microcebus were more likely to engage in stereotypic behaviour than members of other genera. The presence of stereotypic behaviour is also used as a marker of poor welfare (Broom 1986; Honess and Marin 2006; Mallapur and Choudhury 2003). Frustration is one of the manifestations of stereotypy. It results in animals from not being able to perform a species-specific behaviour pattern, or from social deprivation, unavoidable stress or fear, etc. (Tarou et al. 2005). Whereas locomotor stereotypy 
(pacing) and frustration were observed in both lemurs in their original housing, neither was seen during the periods of observation in their new environment after the transport. As one aspect of the environment that has been considered to cause stereotypic behaviour is boredom (Mallapur and Choudhury 2003), the effect of transport and change of the environment may have played a positive role in this case. The new enclosure with its natural soil surface covered with leaves and the presence of trees offered many options for exploration as well as for the satisfaction of speciesspecific needs of black-and-white ruffed lemurs as described by Pereira et al. (1988). However, the frequency of exploratory behaviour did not significantly differ within the monitored period before and after the transport. It was mainly caused by the variation of the frequency of exploration in the days following the transport. While in the first two days after the transport the exploration was almost missing, it rapidly increased later. Because of the short period of observation after the transport, it is not known if it was only a short-term effect of the novelty or if the new environment suited the lemurs better and their abnormal behaviour patterns disappeared altogether.

Behavioural responses to stress are evoked from the underlying complex physiological changes resulting from stress. In our study, the endocrine effect of transport stress was determined using non-invasive assessment of faecal samples, which has become an invaluable tool for studying the behavioural ecology of primates (Behringer and Deschner 2017). The marked increase in FGM concentrations was observed in 1.5 day after the transport. The delay in glucocorticoid excretion is correlated with the transit time of digesta. A comparable lag time was observed in grey mouse lemurs (Microcebus murinus), where the FGM concentrations started to increase $24 \mathrm{~h}$ after exposure to a stress stimulus; the median lag time to peak FGM response was 38 to $50 \mathrm{~h}$ (Hämäläinen et al. 2014). In other primates the concentrations varied, for example, in captive chimpanzees and Barbary macaques, FGM levels were elevated 1-2 days after the stress impulse (Whitten et al. 1998; Heistermann et al. 2006), whereas in marmosets the FGM levels peaked already $7 \mathrm{~h}$ after the stress impulse. In pileated gibbons the peak response was determined from 22 to $55 \mathrm{~h}$ and the levels usually returned to pre-treatment baseline levels by day 5 (Pirovino et al. 2011). The time course of change in FGM levels observed in our study is in agreement with the previous studies indicating that transport of animals results in an short-time increase of FGM levels (Goymann et al. 1999; Terio et al. 1999; Palme et al. 2000; Möstl et al. 2002; Dembiec et al. 2004; Touma and Palme 2005; Laws et al. 2007; Capiro et al. 2014; Keller et al. 2014; Bashaw et al. 2016). The excretion of glucocorticoids into faeces is affected by many factors. Differences among species must be taken into consideration, as the excretion is influenced by the different formations of the digestive tract, ingestion of different kinds of food and different metabolisms (Touma et al. 2004; Palme 2005; Goymann 2012). Our study provides new data on the glucocorticoid response to transport and its progress in black-and-white ruffed lemurs. All EIAs tested were found to be sensitive enough to show stress-related changes in FGM levels in black-and-white ruffed lemurs.

In conclusion, the results of both the behaviour and the adrenocortical activity analysis suggest that despite some presented changes in the monitored indices prior to and after the transport, the transportation under the studied conditions presented only a mild stressor with limited behavioural and glucocorticoid responses.

\section{Acknowledgements}

The authors of this study thank the Olomouc and Brno ZOOs, namely, zoologist Ing. Jitka Vokurková and Ing. Dorota Gremlicová, curator of mammals, for making the research possible. Also many thanks for the advice to Prof. Rupert Palme of the University of Veterinary Medicine, Vienna, and Edith Klobetz Rassam and Elke Leitner for technical assistance with the EIA analysis. 


\section{References}

Baker ML, Gemmell RT 1999: Physiological changes in the brushtail possum (Trichosurus vulpecula) following relocation from Armidale to Brisbane, Australia. J Exp Zool 284: 42-49

Bashaw MJ, Sicks F, Palme R, Schwarzenberger F, Tordiffe ASW, Ganswindt A 2016: Non-invasive assessment of adrenocortical activity as a measure of stress in giraffe (Giraffa camelopardalis). BMC Vet Res 235: 1-13

Behringer V, Deschner T 2017: Non-invasive monitoring of physiological markers in primates. Horm Behav 91: 3-18

Bray J, Samson DR, Nunn ChL 2016: Activity patterns in seven captive lemur species: evidence of cathemerality in Varecia and Lemur catta? Am J Primatol 79: 1-9

Broom DM 1986: Indicators of poor welfare. Br Vet J 142: 524-526

Brundige L, Oleas T, Doumit M, Zanella AJ 1998: Loading techniques and their effect on behavioral and physiological responses of market weight pigs. J Anim Sci 76: 99

Campbell JL, Eisemann JH, Williams CV, Glenn KM 2000: Description of the gastrointestinal tract of five lemur species: Propithecus tattersalli, Propithecus verreauxi coquereli, Varecia variegata, Hapalemur griseus, and Lemur catta. Am J Primatol 52: 133-142

Capiro JM, Stoops MA, Freeman EW, Clawson D, Schook MW 2014: Effects of management strategies on glucocorticoids and behavior in Indian rhinoceros (Rhinoceros unicornis): Translocation and operant conditioning. Zoo Biol 33: 131-143

Cavigelli SA 1999: Behavioural patterns associated with faecal cortisol levels in free-ranging female ring-tailed lemurs, Lemur catta. Anim Behav 57: 935-944

Cavigelli SA, Dubovick T, Levash W, Jolly A, Pitts A 2003: Female dominance status and fecal corticoids in a cooperative breeder with low reproductive skew: Ring-tailed lemurs (Lemur catta). Horm Behav 43: 166-179

CITES 2017. Appendices I, II and III. Available at: https://cites.org/sites/default/files/eng/app/2017/EAppendices-2017-10-04.pdf. Accessed March 10, 2018

Clark FE, Fitzpatrick M, Hartley A, King AJ, Lee T, Routh A, Walker SL, George K 2011: Relationship between behavior, adrenal activity, and environment in zoo-housed Western lowland gorillas (Gorilla gorilla gorilla). Zoo Biol 30: 1-16

Dembiec DP, Snider RJ, Zanella AJ 2004: The effects of transport stress on tiger physiology and behavior. Zoo Biol 23: 335-346

Frigerio D, Dittami J, Möstl E, Kotrschal K 2004: Excreted corticosterone metabolites co-vary with ambient temperature and air pressure in male greylag geese (Anser anser). Gen Comp Endocrinol 137: 29-36

Gould L, Ziegler TE, Wittwer DJ 2005: Effects of reproductive and social variables on fecal glucocorticoid levels in a sample of adult male ring-tailed lemurs (Lemur catta) at the Beza Nahafaly Reserve, Madagascar. Am J Primatol 67: 5-23

Goymann W, Möstl E, Van't Hof T, East ML, Hofer H 1999: Noninvasive fecal monitoring of glucocorticoids in spotted hyenas, Crocuta crocuta. Gen Comp Endocrinol 114: 340-348

Goymann W 2012: On the use of non-invasive hormone research in uncontrolled, natural environments: the problem with sex, diet, metabolic rate and the individual. Methods Ecol Evol 3: 757-765

Grandin T 1997: Assessment of stress during handling and transport. J Anim Sci 75: 249-257

Hämäläinen A, Heistermann M, Fenosoa ZSE, Kraus C 2014: Evaluating capture stress in wild gray mouse lemurs via repeated fecal sampling: method validation and the influence of prior experience and handling protocols on stress responses. Gen Comp Endocrinol 195: 68-79

Heistermann M, Palme R, Ganswindt A 2006: Comparison of different enzymeimmunoassays for assessment of adrenocortical activity in primates based on fecal analysis. Am J Primatol 68: 257-273

Honess PE, Marin CM 2006: Enrichment and aggression in primates. Neurosci Biobehav Rev 30: 413-436

IUCN 2018: Varecia variegata. Available at: http://www.iucnredlist.org/details/22918/0. Accessed March 10, 2018

King T, Rasolofoharivelo T, Chamberlan C 2013: Conserving the critically endangered black-and white ruffed lemur Varecia variegata through integrating ex situ and in situ efforts. Wild Conservation 1: 25-30

Kelley EA, Jablonski NG, Chaplin G, Sussman RW, Kamilar JM 2016: Behavioral thermoregulation in Lemur catta: The significance of sunning and huddling behaviors. J Am Primatol 78: 745-754

Keller KL, Fritz RS, Zoubek CM, Kennedy EH, Cronin KA, Rothwell ES, Serfass TL 2014: Effects of transport on fecal glucocorticoid levels in captive-bred cotton-top tamarins (Sanguinus oedipus). J Pa Acad Sci 88: 84-88

Krief S, Jamart A, Hladik CM 2004: On the possible adaptive value of coprophagy in free-ranging chimpanzees. Primates 45: 141-145

Laws N, Ganswindt A, Heistermann M, Harris M, Harris S, Sherwin C 2007: A case study: Fecal corticosteroid and behavior as indicators of welfare during relocation of an Asian elephant. J Appl Anim Welf Sci 10: 349-358

Malikova M, Voslarova E, Vecerek V 2018: Non-invasive faecal monitoring of stress in captive and free-ranging wild animals. Berl Münch Tierärztl Wechenschr 131: 150-158

Mallapur A, Choudhury BC 2003: Behavioral abnormalities in captive nonhuman primates. J Appl Anim Welf Sci 6: 275-284

Möstl E, Maggs JL, Schrötter G, Besenfelder U, Palme R 2002: Measurement of cortisol metabolites in faeces of ruminants. Vet Res Commun 26: 127-139 
Palme R, Möstl E 1997: Measurement of cortisol metabolites in faeces of sheep as a parameter of cortisol concentration in blood. Z Saugetierkd 62: 192-197

Palme R, Robia C, Baumgartner W, Möstl E 2000: Transport stress in cattle as reflected by an increase in faecal cortisol metabolite concentrations. Vet Rec 146: 108-109

Palme R 2005: Measuring fecal steroids: guidelines for practical application. Ann N Y Acad Sci 1046: 75-80

Pereira ME, Seelingson ML, Macedonia JM 1988: The behavioral repertoire of the black-and-white ruffed lemur, Varecia variegata variegata (Primates: Lemuridae). Folia Primatol 51: 1-32

Pirovino M, Heistermann M, Zimmermann N, Zingg R, Clauss M, Codron D, Kaup FJ, Steinmetz HW 2011: Fecal glucocorticoid measurements and their relation to rearing, behavior, and environmental factors in the population of pileated gibbons (Hylobates pileatus) held in European zoos. Int J Primatol 32: 1161-1178

Porton I 1989: International studbook for the ruffed lemur Varecia variegata. St. Louis: St. Louis Zool Park

Pride RE 2005: Optimal group size and seasonal stress in ring-tailed lemurs (Lemur catta). Behav Ecol 16: 550-560

Rangel-Negrín A, Flores-Escobar E, Chavira R, Canales-Espinosa D, Dias PAD 2014: Physiological and analytical validations of fecal steroid hormone measures in black howler monkeys. Primates 55: 459-465

Sapolsky RM, Romero LM, Munck AU 2000: How do glucocorticoids influence stress responses? Integrating permissive, suppressive, stimulatory, and preparative actions. Endocr Rev 21: 55-89

Shutt K, Setchell JM, Heistermann M 2012: Non-invasive monitoring of physiological stress in the western lowland gorila (Gorilla gorila gorila): validation of fecal glucocorticoid assay and methods for practical application in the field. Gen Comp Endocrinol 179: 167-177

Schwarzenberger F and Brown JL 2013: Hormone monitoring: An important tool for the breeding management of wildlife species. Wien Tierarztl Monatsschr 100: 209-225

Schwitzer C, King T, Robsomanitrandrasana E, Chamberlan C, Rasolofoharivelo T 2013: Integrating ex situ and in situ conservation of lemurs. In: Schwitzer C et al. (Eds) 2013: Lemurs of Madagascar: A Strategy for their conservation 2013-2016. Bristol, UK: IUCN SSC Primate Specialist Group, Bristol Conservation and Science Foundation, and Conservation International, pp. 146-152

Starling AP, Charpentier MJE, Fitzpatrick C, Scordato ES, Drea CHM 2010: Seasonality, sociality, and reproduction: long-term stressors of ring-tailed lemurs (Lemur catta). Horm Behav 57: 76-85

Takeshita RSC, Bercovitch FB, Huffman MA, Kinoshita K 2018: Development and validation of an enzyme immunoassay for fecal dehydroepiandrosterone sulfate in Japanese macaques (Macaca fuscata). Int J Primatol 39: 208-221

Tarou LR, Bloomsmith MA, Maple TL 2005: Survey of stereotypic behavior in prosimians. Am J Primatol 65: 181-196

Terio KA, Citino SB, Brown JL 1999: Fecal cortisol metabolite analysis for noninvasive monitoring of adrenocortical function in the cheetah (Acinonyx jubatus). J Zoo Wildl Med 30: 484-491

Touma CH, Palme R, Sachser N 2004: Analyzing corticosterone metabolites in fecal samples of mice: a noninvasive technique to monitor stress hormones. Horm Behav 45: 10-22

Touma CH, Palme R 2005: Measuring fecal glucocorticoid metabolites in mammals and birds: the importance of validation. Ann N Y Acad Sci 1046: 54-74

Vasey N 2006: Impact of seasonality and reproduction on social structure, ranging patterns, and fission-fusion social organization in red ruffed lemurs. In: Gould L, Sauther ML (Eds). Lemurs: ecology and adaptation. New York: Springer. 275-304

Weingrill T, Willems EP, Zimmermann N, Steinmetz H, Heistermann M 2011: Species-specific patterns in fecal glucocorticoid and androgen levels in zoo-living orangutans (Pongo spp.). Gen Comp Endocrinol 172: 446-457

Whitten PL, Stavisky R, Aureli F, Russel E 1998: Response of fecal cortisol to stress in captive chimpanzees (Pan troglodytes). Am J Primatol 44: 57-69

Zar JH 1999. Biostatistical Analysis. $4^{\text {th }}$ edn. Prentice-Hall, Inc., New Jersey, 663 p. 